\title{
Engineering of boron-induced dislocation loops for efficient room-temperature silicon light-emitting diodes
}

\author{
M. Milosavljevića ${ }^{a}$ \\ Advanced Technology Institute, School of Electronics and Physical Sciences, University of Surrey, \\ Guildford, Surrey, GU2 7XH, United Kingdom \\ G. Shao \\ School of Engineering, University of Surrey, Guildford, Surrey, GU2 7XH, United Kingdom \\ M. A. Lourenco, R. M. Gwilliam, and K. P. Homewood \\ Advanced Technology Institute, School of Electronics and Physical Sciences, University of Surrey, \\ Guildford, Surrey, GU2 7XH, United Kingdom
}

(Received 13 September 2004; accepted 7 January 2005; published online 23 March 2005)

\begin{abstract}
We have studied the role of boron ion energy in the engineering of dislocation loops for silicon light-emitting diodes (LEDs). Boron ions from 10 to $80 \mathrm{keV}$ were implanted in (100) Si at ambient temperature, to a constant fluence of $1 \times 10^{15} \mathrm{ions} / \mathrm{cm}^{2}$. After irradiation the samples were annealed for $20 \mathrm{~min}$ at $950^{\circ} \mathrm{C}$ by rapid thermal annealing. The samples were analyzed by transmission electron microscopy and Rutherford backscattering spectroscopy. It was found that the applied ion implantation/thermal processing induces interstitial perfect and faulted dislocation loops in $\{111\}$ habit planes, with Burgers vectors $a / 2\langle 110\rangle$ and $a / 3\langle 111\rangle$, respectively. The loops are located around the projected ion range, but stretch in depth approximately to the end of range. Their size and distribution depend strongly on the applied ion energy. In the $10 \mathrm{keV}$ boron-implanted samples the loops are shallow, with a mean size of $\sim 30 \mathrm{~nm}$ for faulted loops and $\sim 75 \mathrm{~nm}$ for perfect loops. Higher energies yield buried, large, and irregularly shaped perfect loops, up to $\sim 500 \mathrm{~nm}$, coexisting with much smaller faulted loops. In the latter case much more $\mathrm{Si}$ interstitials are bounded by the loops, which are assigned to a higher supersaturation of interstitials in as-implanted samples, due to separated Frenkel pairs. An interesting phenomenon was found: the perfect loops achieved a steady-state maximum size when the ion energy reached $40 \mathrm{keV}$. Further increase of the ion energy only increased the number of these large loops and made them bury deeper in the substrate. The results of this work contribute to laying a solid ground in controlling the size and distribution of dislocation loops in the fabrication of silicon LEDs. (C) 2005 American Institute of Physics. [DOI: $10.1063 / 1.1866492]$
\end{abstract}

\section{INTRODUCTION}

Achieving efficient light emission from silicon is a challenge for future progress in ultralarge-scale integration (ULSI) technology, where optical interconnects will be an ultimate solution as the devices become smaller and faster. Numerous methods were investigated to produce light emitters from silicon and silicon-based materials, the disadvantage of pure silicon being in its inefficient light emission due to the indirect nature of the electronic band gap. One of earlier approaches demonstrated a light-emitting diode (LED) that contained precipitates of $\beta \mathrm{FeSi}_{2}$ embedded in silicon, ${ }^{1}$ the silicide itself being a direct band-gap semiconductor. ${ }^{2,3}$ However, despite being efficient at low temperatures the emission due to $\beta \mathrm{FeSi}_{2}$ quenched to low values at room temperature. Recent efforts yielded a discovery of an all-silicon LED, produced by dislocation engineering, which involves ion implantation and subsequent heat treatments. ${ }^{4}$ The essence of this recent method is a controlled introduction of dislocation loops which create a strain field

\footnotetext{
a) Author to whom correspondence should be addressed; on leave from VINČA Institute of Nuclear Sciences, Belgrade, Serbia and Montenegro; electronic mail: m.milosavljević@ surrey.ac.uk
}

that modifies the silicon band gap, thus preventing nonradiative and enhancing radiative transitions of electrical carriers. Indeed, light-emitting diodes efficient at room temperature were fabricated by implantation of boron into silicon, where the role of boron was both as a dopant to form a $p-n$ junction, as well as a means to introduce dislocation loops. $^{4-6}$

Dislocation loops and other linear defects were studied intensively as a residual damage after ion implantation doping of silicon. In those studies they were treated as unwanted effects, causing enhanced diffusion of dopants and/or failure of fabricated devices. Special attention was given to transient-enhanced diffusion (TED) of dopants, which happens during the initial stages of postimplantation annealing, and it was correlated to the evolution of linear and planar defects during annealing at different temperatures and times. $^{7-11}$ Systematic studies ${ }^{12-20}$ of nucleation, growth, and transformation of these defects, performed with the aim to understand and possibly minimize or eliminate their effects, are now useful if they are to be produced deliberately. As ion implantation increases local density, the induced defects are extrinsic in nature, and the dislocation loops that are formed are interstitial. In a classification introduced by Jones et al., ${ }^{14}$ 
there are five categories of defects that can be created in silicon by ion implantation, depending on the ion fluence, mass, and energy. Crystal damages nominated as category I and II in this classification are most interesting for a controlled formation of dislocation loops. In the first case no amorphous layer is formed in Si during ion implantation, but the necessary fluence for defects to appear is above a critical value of $\sim 2 \times 10^{14}$ ions $/ \mathrm{cm}^{2}$, irrelevant of the ion species. ${ }^{14}$ Defects formed during annealing at $700-1000{ }^{\circ} \mathrm{C}$ consist mainly of $\{113\}$ rodlike defects, faulted Frank dislocation loops, and perfect dislocation loops located around the projected ion range in Si. Both faulted and perfect dislocation loops are interstitial, having $\{111\}$ habit planes, and Burgers vectors $a / 3\langle 111\rangle$ and $a / 2\langle 110\rangle$, respectively. ${ }^{12-14}$ The majority of studies reported in the literature are concerned with category II damage, when ion implantation forms an amorphous layer in silicon. Preamorphization of silicon (prior to implantation of boron) was carried out with heavier ions, such as $\mathrm{Si}$ or Ga. ${ }^{10,11}$ The defects that form during annealing are so called "end of range" (EOR) defects because they originate from the initial amorphous/crystalline interface. For similar heat treatments they consist also of rodlike defects and faulted and perfect dislocation loops, but they are located deeper in the substrate than category I defects, and the dislocation loops are considerably smaller.

In this paper we present a study of the nature and distribution of dislocation loops in silicon LED structures fabricated by boron ion implantation in the energy range from 10 to $80 \mathrm{keV}$. After implantation the $\mathrm{Si}$ samples were annealed at $950{ }^{\circ} \mathrm{C}$ for $20 \mathrm{~min}$, the conditions that have been found to induce dislocation loop structures that provide efficient light emission at room temperature. ${ }^{5,6,21}$ The main analysis of the samples was performed by transmission electron microscopy (TEM), and we also used Rutherford backscattering spectroscopy (RBS) to study the damage distribution in silicon. As there was no amorphizaton of the $\mathrm{Si}$ substrates by ion irradiation, the induced defects belong to category I damage, and consist of perfect and faulted dislocation loops. The loops are located around the projected ion range, though they stretch in depth approximately to the end of ion range.

\section{EXPERIMENTAL DETAILS}

The base material used for these experiments and for fabrication of light-emitting diodes were 4-in. $n$-type (100) silicon wafers (phosphorous doped, 2-7 $\Omega \mathrm{cm}$ ). They were implanted with boron ions, using a Danfysik ion implanter. The ion energy was varied in the range from 10 to $80 \mathrm{keV}$, in steps of $10 \mathrm{keV}$, while the implanted fluence was kept constant at $1 \times 10^{15}$ ions $/ \mathrm{cm}^{2}$. The corresponding projected ion range $R_{p}$ varied from $39 \mathrm{~nm}$ for $10 \mathrm{keV}$ to $275 \mathrm{~nm}$ for $80 \mathrm{keV}$, as deduced by the TRIM code. ${ }^{22}$ During implantation the wafers were mounted on a carousel holder held at ambient temperature, and the beam current was held below $1 \mu \mathrm{A} / \mathrm{cm}^{2}$ to avoid beam heating of the targets. After ion implantation all the samples were annealed under the same conditions, in a nitrogen ambient for $20 \mathrm{~min}$ at $950{ }^{\circ} \mathrm{C}$, by

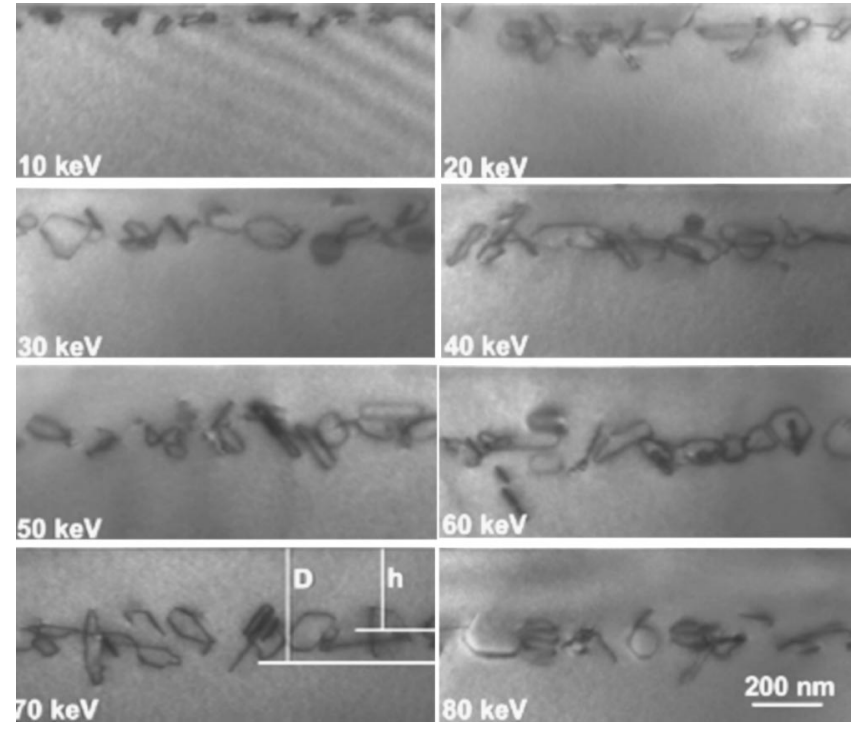

FIG. 1. Bright-field XTEM images taken along [110] Si of the samples implanted with boron at $10-80 \mathrm{keV}$.

rapid thermal annealing (RTA). Fabrication of light-emitting diodes was then completed by deposition of front and back contacts, as described previously. ${ }^{4-6}$

For TEM analysis we prepared both cross-sectional (XTEM) and plan-view specimens of all the samples, using low-angle argon ion-beam thinning at $5 \mathrm{keV}$. The analysis was done on Philips EM 400 T and CM 200 electron microscopes, operated at 120 and $200 \mathrm{kV}$, respectively. Since our samples contained both perfect and faulted dislocation loops with Burgers vectors $a / 2\langle 110\rangle$ and $a / 3\langle 111\rangle$, in order to observe all of them in plan-view specimens we used an offaxis four-beam imaging condition, which gives better contrast than the symmetrical on-axis [001] imaging condition, as proposed by Pan et al. ${ }^{17,18}$ For determining the Burgers vectors of dislocation loops, we used $\{220\}$ and $\{004\}$ twobeam conditions. Standard trace analysis techniques have been used to determine the habit plane and the intrinsic/ extrinsic nature of the loops. ${ }^{23}$ In XTEM samples we used the [110] zone axis to image the loops. RBS analysis was done with a $1.5-\mathrm{MeV} \mathrm{He}^{+}$beam and two detectors positioned at $147^{\circ}$ and $166^{\circ}$ backscattering angles. Channeling technique was used to study the damage, the yield being compared to virgin silicon.

\section{RESULTS}

The results of the TEM analysis have revealed that after boron implantation and the applied annealing, $20 \mathrm{~min}$ at $950{ }^{\circ} \mathrm{C}$, all the samples contain only extrinsic dislocation loops. Other possible linear defects were dissolved at these annealing conditions. The dislocation loops have $\{111\}$ habit planes and are either perfect or faulted. Their depth distribution, shape, and size depend strongly on the applied ion energy.

A series of bright-field XTEM images taken from the samples implanted with boron at energies ranging from 10 to $80 \mathrm{keV}$ is presented in Fig. 1. All images were taken along the [110] Si zone axis. We can see oval shapes due to 


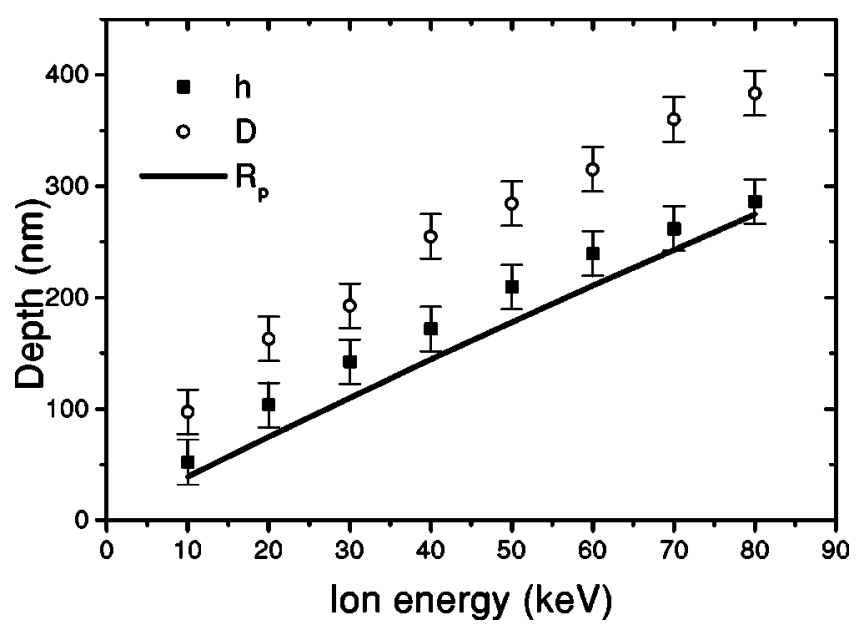

FIG. 2. Dependence of the middle position of the loops $h$, maximum depth of the loops from the surface $D$ and of the ion range $R_{p}$ upon implantation energy.

circular dislocation loops inclined to the imaging direction, and trace lines of loops lying in planes parallel to [110], but also some irregular shapes originating from large irregularly shaped perfect loops as will be seen from the plan-view images. As the ion energy is increased, the loops enlarge in size and are buried deeper in the Si substrate. In Fig. 2 we have plotted the measured values of $h$, the middle position of the loops, and $D$, the maximum depth of the loops from the surface (indicated in Fig. 1) as a function of ion energy, as well as the values of $R_{p}$ obtained from TRIM. It is seen that, within the experimental error, the loops are located around the projected ion range, but as they are relatively large in size, their inner edges spread much deeper.

Plan-view images of selected samples are presented in Fig. 3. They were taken under the off-axis four-beam imag-

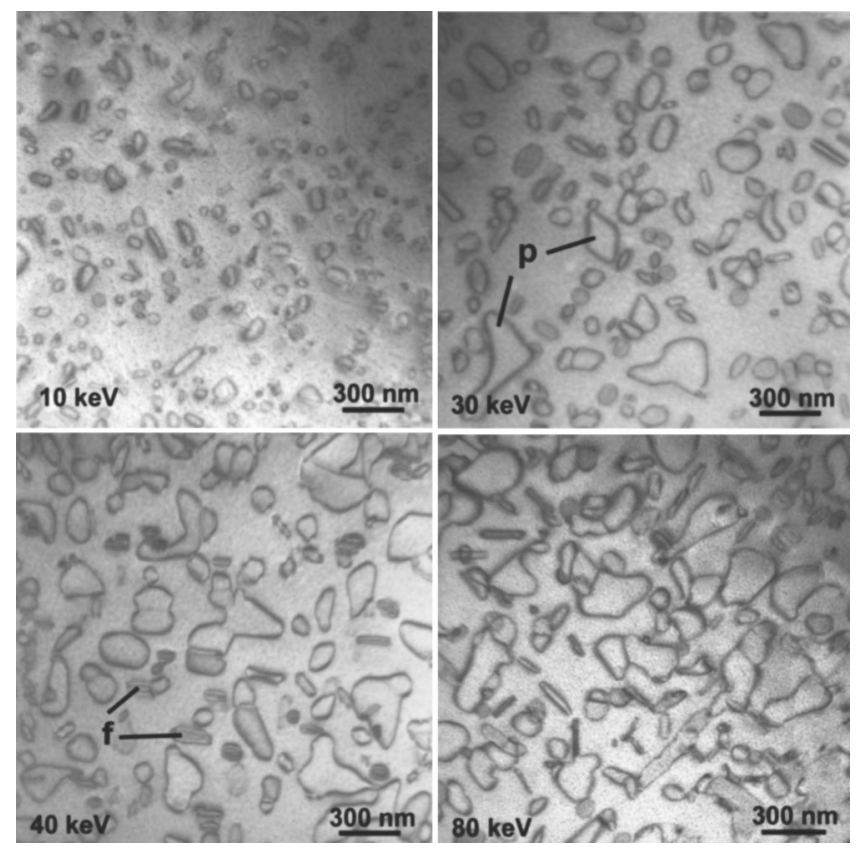

FIG. 3. Bright-field plan-view images taken under off-axis four-beam imaging conditions near [001] Si of the samples implanted at different boron energies. The markers indicate perfect (p) and faulted (f) dislocation loops.

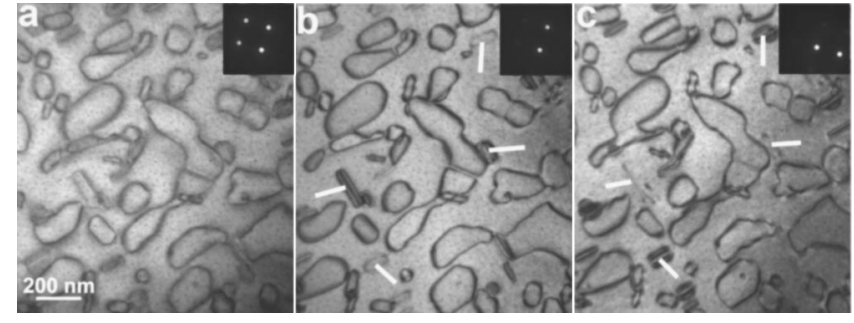

FIG. 4. Disappearing and reappearing of $a / 3\langle 111\rangle$ loops, using different $\{220\}$ two-beam conditions. Some of these faulted dislocation loops are pointed by the markers.

ing condition when the pole of the [001] pattern is tilted to overlap with the $\{020\}$ beam, allowing all loops to be observed with good contrast. The samples were thinned from the back, and we chose thicker areas for analysis, where the loops were not partly cut off by thinning. The plan-view images illustrate how the loops develop with increasing the ion energy. In the $10 \mathrm{keV}$ implanted sample the loops appear the smallest, with a mean size of $\sim 30 \mathrm{~nm}$ for faulted loops and $\sim 75 \mathrm{~nm}$ for perfect loops, although some perfect loops grow up to $\sim 200 \mathrm{~nm}$. For higher implantation energies perfect loops grow much larger. Examples of perfect and faulted dislocation loops are indicated by markers in Fig. 3. For the ion energy of $40 \mathrm{keV}$ the maximum size of perfect dislocation loops tends to saturate at $\sim 500 \mathrm{~nm}$, and for higher energies only the number of these large loops increases. Apart from large perfect loops, in high-energy implanted samples we still observe much smaller faulted loops. In the studies of EOR loops in preamorphized silicon, perfect dislocation loops were also referred to as "prismatic," as they are usually elongated along the $\langle 110\rangle$ directions, or can have rectangular shapes with edges parallel to these directions. ${ }^{18,24}$ However, the EOR loops are much smaller (typically a few tens of nanometers, both perfect and faulted) than the loops that we have produced. In our case we also observe some prismatically shaped loops, as shown for the sample implanted at $30 \mathrm{keV}$, but generally they are irregularly shaped. This appearance is probably due to a form of ripening, when perfect loops join with other such loops that have started to grow in other directions.

In Fig. 4 we illustrate the analysis of a sample implanted at $40 \mathrm{keV}$, showing how dislocation loops can be distinguished among themselves and their Burgers vectors determined. We used the $\mathbf{b} \cdot \mathbf{g}=0$ rule with two-beam imaging conditions typical for studying diffraction contrast, where $\mathbf{b}$ is the Burgers vector of the loops and $\mathbf{g}$ a vector in the reciprocal space, normal to the reflecting plane in the real space. Under such orientation of the sample, the images of the loops that satisfy this condition disappear, or they only show a weak residual contrast. For the off-axis four-beam condition used in Fig. 4(a), all dislocation loops can be seen. In this case the diffraction pattern consists of the transmitted beam, two adjacent $\{220\}$ reflections, and one $\{004\}$ reflection diagonal to the transmitted beam. However, for two-beam conditions, using $\{220\}$ reflections as in Figs. 4(b) and 4(c), some of the loops disappear. These are faulted Frank loops with 


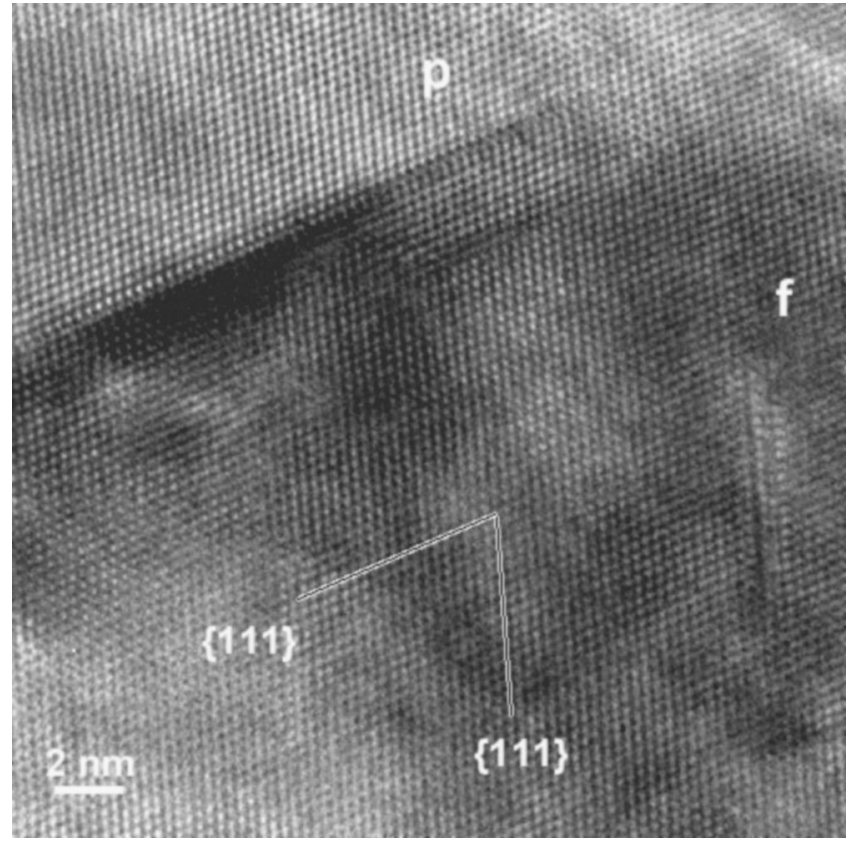

FIG. 5. High-resolution XTEM image of a Si sample implanted with 20 $-\mathrm{keV}$ boron, aligned in the [110] direction. The Labels denote a perfect (p) and a faulted (f) dislocation loop.

Burgers vectors $a / 3\langle 111\rangle$. From a similar analysis, using $\{004\}$ reflections, we have determined the Burgers vector of the larger perfect loops as $a / 2\langle 110\rangle$.

By trace analysis (not presented here) we have determined that both types of loops are interstitial and that their habit planes are $\{111\}$. Direct evidence is presented by a high-resolution XTEM image in Fig. 5, taken along [110] from a sample implanted at $20 \mathrm{keV}$. The image shows cross sections of two neighboring dislocation loops, lying in two different $\{111\}$ planes parallel to the e-beam. The shorter loop (f) is seen as a whole, while from the longer loop (p) we only see one end, as it stretches much further to the left of the view field. Both loops induce stress in the surrounding Si lattice, which is seen as an increased darkness that spreads laterally, parallel to the loops. Using our operating condition (Scherzer defocused), the rows of white spots in the image originate from empty spaces in the crystal lattice, and they are blocked where the loops are positioned. Also, the smaller loop is a faulted Frank dislocation loop as it exhibits a stacking fault sequence, and the longer one is a perfect dislocation loop.

Damage distribution in the as-implanted samples and depth distribution of dislocation loops in the annealed samples were analyzed by RBS. In Fig. 6 we present the RBS channeling spectra of the as-implanted and annealed samples, after boron irradiation to $1 \times 10^{15} \mathrm{ions} / \mathrm{cm}^{2}$, at 20 and $80 \mathrm{keV}$. The channeling was done in the [001] Si direction, and for comparison we included a channeled spectrum of virgin $\mathrm{Si}$. In the as-implanted samples we can see an increased yield compared to virgin $\mathrm{Si}$, located immediately behind the surface Si peak. This comes from radiation damage, i.e., mainly from $\mathrm{Si}$ atoms that are displaced from their lattice positions in collision cascades induced by the impact ions. The damage spreads much deeper in the sample im-

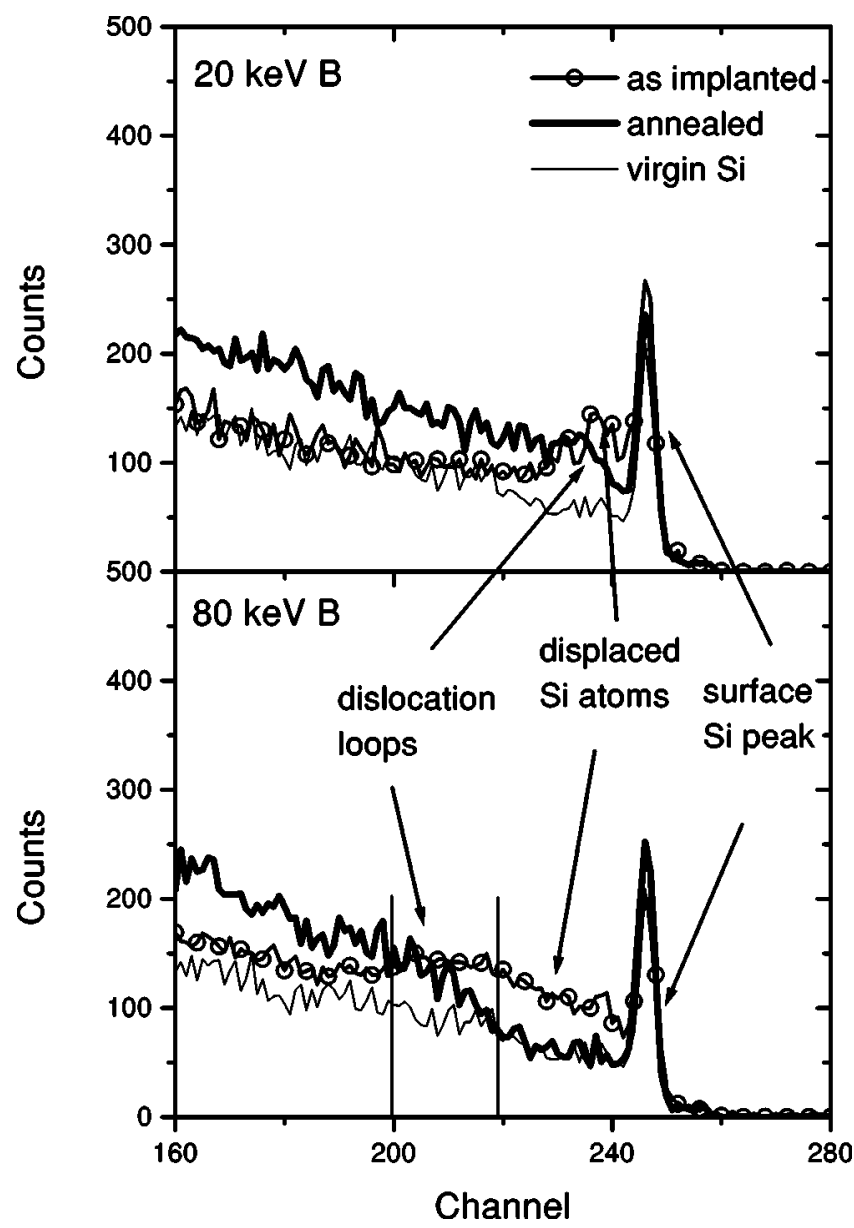

FIG. 6. RBS channeling spectra of as-implanted and annealed Si samples, with boron ions at 20 and $80 \mathrm{keV}$.

planted at higher energy, and at the end of the damaged region the backscattering yield drops practically to the level for virgin $\mathrm{Si}$. The spectra taken from the annealed samples are different, and if we compare to the XTEM analysis in Fig. 1, we can find similarities. The surface region of the samples has recrystallized and hence the RBS yield is at the channeling level of single-crystal Si. Gradual increase of RBS yield with respect to virgin $\mathrm{Si}$ begins at a depth where the dislocation loops start to appear, and advances to their inner edge in the Si substrate. Finally, the yield from the deeper region of $\mathrm{Si}$ does not drop as in the case of the as-implanted samples, but the analyzing beam remains partly dechanneled by dislocation loops. Thus, from the RBS spectra it was possible to determine the depth and the thickness of the layer that contains dislocation loops. The mean depth values of the loops that were calculated from the RBS spectra are in good agreement with those obtained from the TEM analysis (Fig. 2 ) and are close to the $R_{p}$ values obtained by TRIM.

From the plan-view TEM analysis we have determined the density of dislocation loops and the number of trapped $\mathrm{Si}$ interstitials as a function of boron implantation energy. The total analyzed area of each sample was $4 \times 4 \mu \mathrm{m}^{2}$, which contained up to $\sim 1000$ loops for the highest population. A plot of the loop density (total density of loops and density of faulted loops) is shown in Fig. 7. It is seen that the loop density drops with increasing ion energy, but this does not 


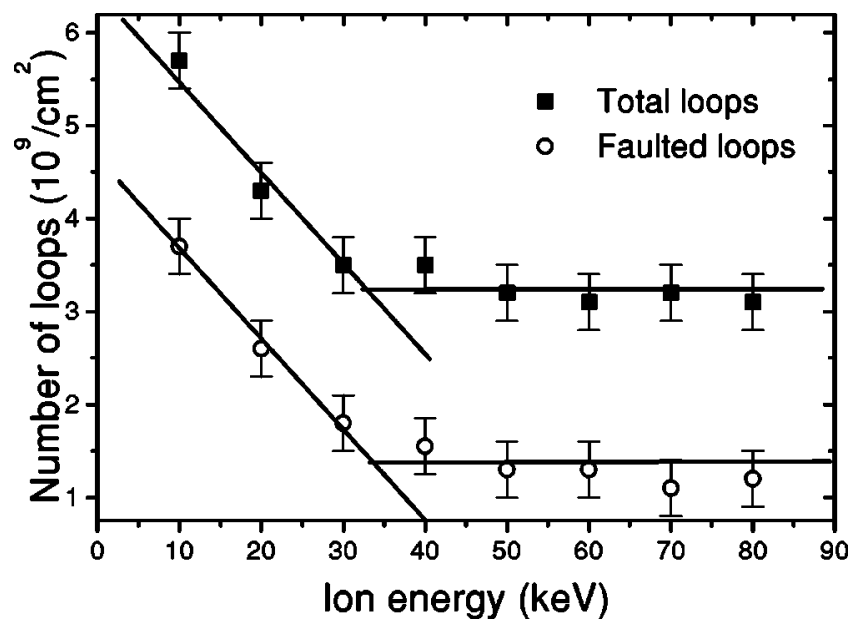

FIG. 7. Total number of dislocation loops and the number of only faulted loops as a function of ion implantation energy.

mean that the number of $\mathrm{Si}$ atoms trapped in the loops decreases, as at the same time they increase in size. Two stages are present in these plots, one from 10 to $40 \mathrm{keV}$ and a second from 40 to $80 \mathrm{keV}$. The borderline at $40 \mathrm{keV}$ coincides with the stage when perfect dislocation loops saturate in size and the large loops only increase in number. This supports the argument that for higher ion energies, when more interstitials are generated in the Si substrate, initially isolated perfect loops tend to coalesce in larger loops. The density of faulted loops also decreases because more Si interstitials are trapped in the perfect loops. The number of Si atoms trapped by the loops increases as a function of ion energy, as plotted in Fig. 8(a). To calculate this we have used the mean size of the loops and their density, and the atomic density of $\mathrm{Si}$ in $\{111\}$ planes $\left(1.57 \times 10^{15}\right.$ at. $\left./ \mathrm{cm}^{2}\right)$. An estimated error here is about $20 \%$ due to approximating the loops to a circular shape, and this adds up to the error in determining the loop density. Nevertheless, a similar trend was found for the number of displaced $\mathrm{Si}$ atoms in the as-implanted samples from the RBS analysis, which is plotted in Fig. 8(b). The number of displaced $\mathrm{Si}$ atoms was determined with respect to the random level, first by normalizing and subtracting the virgin Si spectrum from the channeled spectra of the as-implanted samples and integrating the obtained damage peak.

\section{DISCUSSION}

The engineered dislocation loops belong to category I defects, as the damage induced in silicon by ion implantation is far below the amorphous level, but the applied fluence was sufficient to produce the necessary amount of interstitials to form dislocation loops upon annealing. It was found that the number of displaced silicon atoms in the as-implanted samples is about 2 orders of magnitude higher than the number of atoms trapped in dislocation loops in the annealed samples (Fig. 8). During postimplantation annealing the majority of implantation-induced interstitials and vacancies recombine, and only about $2 \%$ of interstitials are left over to contribute to extrinsic dislocation loops. Monte Carlo simulations of ion implantation infer that vacancies are created closer to the surface, ${ }^{20}$ where the implanted ions have suffi-

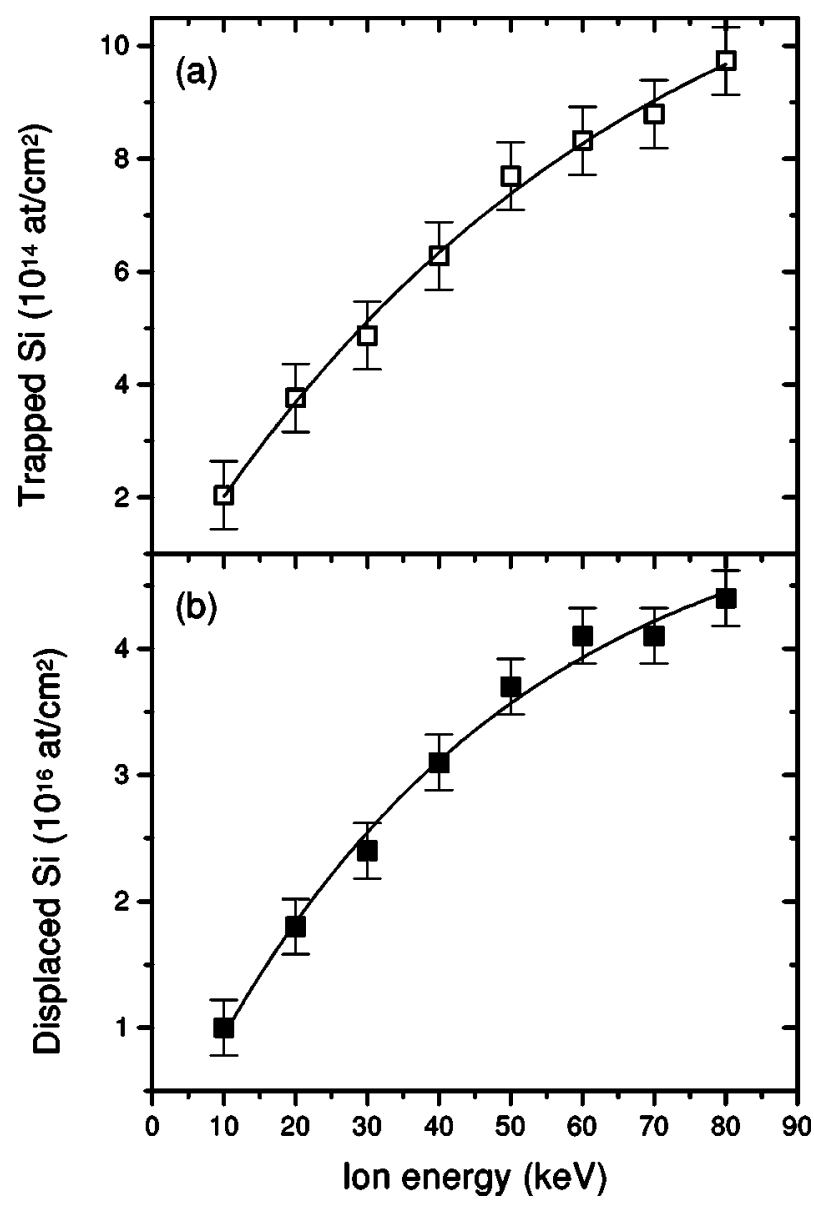

FIG. 8. Number of Si atoms trapped by dislocation loops (a), calculated from the TEM analysis, and the number of originally displaced Si atoms (b) in the as-implanted samples, deduced from the RBS analysis.

cient energy to displace the host atoms from their lattice sites. Interstitials build up deeper in the substrate, typically in a depth region spreading from the projected ion range $R_{p}$ to the end of range, where primary and secondary recoils come to rest. Vacancies that are created near the surface and interstitials located deeper in the substrate are so-called separated Frenkel pairs. Also, due to the substitutional boron dopant being implanted, there is a net increase in the number of atoms around the projected ion range that can be incorporated in the host lattice. During annealing some of the vacancies may diffuse to the surface, leaving an excess number of interstitials buried deeper in the substrate. Furthermore, the electrically activated boron preferentially occupies substitutional sites in the Si crystal lattice. Hence, there is a supersaturation of Si interstitials that cannot recombine and this is the reason why category I defects are extrinsic. It is still a question of argument whether the increased local concentration due to implanted species or separated Frenkel pairs are a dominant source of interstitials, but it seems that both have a contribution. Evolution from supersaturated interstitials to an array of perfect dislocation loops as a function of annealing has been studied extensively. A model proposed by Tan, ${ }^{25}$ and also described in detail by Jones et al., ${ }^{14}$ for category I defects is based on numerous observations. In brief, point defects first coalesce in intermediate defect configurations, consisting of small clusters $(\sim 2 \mathrm{~nm})$, rodlike defects, and 
$\{113\}$ stacking faults. Upon annealing at temperatures up to $700{ }^{\circ} \mathrm{C}$ rodlike defects were found to grow, ${ }^{12}$ but at higher temperatures they dissolve and extrinsic faulted dislocation loops begin to grow. After the rodlike defects have dissolved, the remaining defects consist of faulted dislocation loops and dipoles. With further increase of annealing temperature, these faulted loops and dipoles unfault through nucleation of Shockley partial dislocations. Finally at temperatures from 900 to $1000^{\circ} \mathrm{C}$ all faulted loops are unfaulted, and a layer of perfect dislocation loops is formed. ${ }^{13,14}$

Our samples were annealed for $20 \mathrm{~min}$ at $950{ }^{\circ} \mathrm{C}$ and we have found a mixture of perfect and faulted dislocation loops. We also find that the mean size and density of the loops changes with the applied ion energy. Furthermore, we have implanted the same boron fluence of 1 $\times 10^{15}$ ions $/ \mathrm{cm}^{2}$, but the number of $\mathrm{Si}$ atoms trapped in the dislocation loops depends strongly on the applied ion energy. Obviously, at higher energies more $\mathrm{Si}$ interstitials are produced and correspondingly more of them become trapped in dislocation loops (Fig. 8). For the implantation energy of $80 \mathrm{keV}$ the number of $\mathrm{Si}$ interstitials bounded by the loops is close to the value of the implanted ion fluence. Dislocation loops are buried deeper in $\mathrm{Si}$ with increasing ion energy, their mean depth being around the projected ion range, but their inner edge stretches much deeper in the substrate. By comparing the RBS channeled spectra from the as-implanted and annealed samples, it is seen that the loops are located towards the inner edge of the initial damaged zone. The deeper edge of the loops coincides with the end of the damaged zone of the as-implanted samples, as more clearly seen for the sample implanted at $80 \mathrm{keV}$. This implies that the loops stretch approximately to the end of range of the implanted ions, and can be attributed to the contribution of separated Frenkel pairs in the initial supersaturation of interstitials. The number of large irregularly shaped perfect loops increases with the ion energy. Here we cannot use directly the term "ripening," as we were not extending the annealing time or increasing the temperature, but it can be said that it is due to a higher starting concentration of $\mathrm{Si}$ interstitials. The initial neighboring loops are much closer to each other and can more easily coalesce to form large irregularly shaped loops upon further annealing. An interesting phenomenon found in our studies is that for the ion energy from 40 to $80 \mathrm{keV}$ the maximum size of the loops does not change. With increasing ion energy they just increase in number and are buried deeper in the substrate.

In Ref. 19 Cristiano et al. have calculated and plotted the formation energy for both faulted and perfect dislocation loops as a function of their size. It was shown that faulted loops have a lower formation energy and are therefore more energetically stable up to a diameter of $80 \mathrm{~nm}$, while perfect loops are energetically more stable for larger diameters. Our results are in reasonable agreement with these calculations. The mean diameter of faulted loops that we have found ranges from $\sim 30 \mathrm{~nm}$ for $10 \mathrm{keV}$ to $\sim 90 \mathrm{~nm}$ for $80 \mathrm{keV}$ boron ion energy.

\section{CONCLUSIONS}

The evolution of dislocation loops in Si LED structures was studied as a function of boron ion implantation energy. Although we have used the same ion fluence and the same thermal treatment throughout the experiments, it was demonstrated how the dislocation loops can be engineered. By altering the ion energy it is possible to vary the depth, size, and the density of dislocation loops.

The applied ion irradiation and thermal processing produce a layer of perfect and faulted dislocation loops at a depth around the projected ion range, stretching to the end of range. For a low ion energy of $10 \mathrm{keV}$, the loops are shallower and smaller, with a mean size of $\sim 30$ and $\sim 75 \mathrm{~nm}$ for faulted and perfect loops, respectively, and have a denser population. Higher ion energies induce buried, large, and irregularly shaped perfect dislocation loops, up to $\sim 500 \mathrm{~nm}$, coexisting with much smaller faulted loops. We have found that the maximum size of the loops saturates for the boron ion energy of $40 \mathrm{keV}$, and with further increase of the ion energy they are only buried deeper in the Si substrate. This provides a possibility to tailor a device structure by positioning the loops at a desired depth.

The results are significant for dislocation engineering of silicon LEDs, in offering a possibility to design the loop mean size and density, and their location with respect to the $p-n$ junction. The method can be combined with other processing, such as etching, or different ion irradiation/ annealing parameters can be used, to further adjust the appearance and distribution of dislocation loops, and hence influence the corresponding electroluminescence efficiency of silicon LEDs.

${ }^{1}$ D. Leong, M. Harry, K. J. Reeson, and K. P. Homewood, Nature (London) 387, 686 (1997).

${ }^{2}$ M. C. Bost and J. E. Mahan, J. Appl. Phys. 58, 2696 (1985).

${ }^{3}$ Z. Yang, K. P. Homewood, M. S. Finney, M. A. Harry, and K. J. Reeson, J. Appl. Phys. 78, 1958 (1995).

${ }^{4}$ W. L. Ng, M. A. Lourenco, R. M. Gwilliam, S. Ledain, G. Shao, and K. P. Homewood, Nature (London) 410, 192 (2001).

${ }^{5}$ M. A. Lourenco, M. S. A. Siddiqui, R. M. Gwilliam, G. Shao, and K. P. Homewood, Physica E (Amsterdam) 16, 376 (2003).

${ }^{6}$ M. A. Lourenco, M. S. A. Siddiqui, G. Shao, R. M. Gwilliam, and K. P. Homewood, Phys. Status Solidi A 201, 239 (2004).

${ }^{7}$ N. E. B. Cowern, K. T. F. Janssen, and H. F. F. Jos, J. Appl. Phys. 68, 6191 (1990).

${ }^{8}$ C. Bonafos, A. Claverie, D. Alquier, C. Bergaud, A. Martinez, L. Laanab, and D. Mathiot, Appl. Phys. Lett. 71, 365 (1997).

${ }^{9}$ L. S. Robertson, M. E. Law, K. S. Jones, L. M. Rubin, J. Jackson, P. Chi, and D. S. Simons, Appl. Phys. Lett. 75, 3844 (1999).

${ }^{10}$ P. A. Stolk, H.-J. Gossmann, D. J. Eaglesham, and J. M. Poate, Nucl. Instrum. Methods Phys. Res. B 96, 187 (1995).

${ }^{11}$ A. Claverie, L. F. Giles, M. Omri, B. de Mauduit, G. Ben Assayag, and D. Mathiot, Nucl. Instrum. Methods Phys. Res. B 147, 1 (1999).

${ }^{12}$ W.-K. Wu and J. Washburn, J. Appl. Phys. 48, 3747 (1977).

${ }^{13}$ J. J. Comer, Radiat. Eff. 36, 57 (1978).

${ }^{14}$ K. S. Jones, S. Prussin, and E. R. Weber, Appl. Phys. A: Solids Surf. 45, 1 (1988).

${ }^{15}$ B. de Mauduit, L. Laanab, C. Bergaud, M. M. Faye, A. Martinez, and A. Claverie, Nucl. Instrum. Methods Phys. Res. B 84, 190 (1994).

${ }^{16}$ R. D. Goldberg, T. W. Simpson, I. V. Mitchell, P. J. Simpson, M. Prikryl, and G. C. Weatherly, Nucl. Instrum. Methods Phys. Res. B 106, 216 (1995).

${ }^{17}$ G. Z. Pan, K. N. Tu, and S. Prussin, Appl. Phys. Lett. 68, 1654 (1996).

${ }^{18}$ G. Z. Pan and K. N. Tu, J. Appl. Phys. 82, 601 (1997).

${ }^{19}$ F. Cristiano, J. Grisolia, B. Colombeau, M. Omri, B. de Mauduit, A. Cla- 
verie, L. F. Giles, and N. E. B. Cowern, J. Appl. Phys. 87, 8420 (2000).

${ }^{20}$ A. Claverie, B. Colombeau, B. de Mauduit, C. Bonafos, X. Hebras, G. Ben Assayag, and F. Cristiano, Appl. Phys. A: Mater. Sci. Process. 76, 1025 (2003).

${ }^{21}$ M. Milosavljević, M. A. Lourenco, M. S. A. Siddiqui, G. Shao, R. M Gwilliam, and K. P. Homewood, Inst. Phys. Conf. Ser. 179, 99 (2004).

${ }^{22}$ J. F. Ziegler, J. P. Biersak, and U. Littmark, The Stopping and Range of
Ions in Solids (Pergamon, New York, 1985).

${ }^{23} \mathrm{~J}$. W. Edington, Interpretation of Transmission Electron MocrographsPhilips Technical Library 3 (MacMillan, London, 1975).

${ }^{24}$ G. Z. Pan, K. N. Tu, and A. Prussin, J. Appl. Phys. 81, 78 (1997).

${ }^{25}$ T. Y. Tan, in Defects in Semiconductors, edited by J. Narayan and T. Y. Tan (North-Holland, Amsterdam, 1981), pp. 163-172. 